\title{
Dielectric Relaxation and Molecular Motion in the Chiral Main-Chain Liquid Crystalline Copolyester, BB-4*(2-Me)/BB-6
}

\author{
Takeo Furukawa, Osamu Uchinokura, Yoshiyuki TaKaHashi, \\ Masatoshi Tokita*, Kensuke OsadA*, and Junji Watanabe* \\ Department of Chemistry, Faculty of Science, Science University of Tokyo, \\ 1-3 Kagurazaka, Shinjuku, Tokyo 162-8601, Japan \\ * Department of Polymer Chemistry, Faculty of Engineering, Tokyo Institute of Technology, \\ 2-12-1 O-okayama, Meguro, Tokyo 152-8552, Japan
}

(Received June 22, 1999)

\begin{abstract}
Broad-band dielectric relaxation spectroscopy has been used to examine molecular motions in a mainchain liquid crystalline copolyester BB-4*(2-Me)/BB-6 consisting of a biphenyldicarboxylic acid and equimolar mixture of chiral 2-methylbutanediol and hexanediol. From 60 to $140^{\circ} \mathrm{C}$ where molecules assume a chiral smectic C phase, BB-4* $(2$ Me)/BB-6 exhibits a Goldstone mode process whose relaxation strength rapidly decreases with increasing dc bias field. Combining dielectric relaxation time and strength allows estimate of interlayer rotational viscosity which is $10-100$ times larger than that of low molecular weight liquid crystals reflecting methylene chains connecting smectic layers. Decreasing temperature leads to two additional processes, Vogel-Fulcher type and Arrhenius types. These processes are analogous to segmental and local mode motion characteristic of non-crystalline polymers. The former defines a $T_{\mathrm{g}}$ of 15 ${ }^{\circ} \mathrm{C}$. The coexistence of the Goldstone mode and segmental mode indicates that BB- $4^{*}(2-\mathrm{Me}) / \mathrm{BB}-6$ possesses both orientational order forming liquid crystalline phases and disorder required to undergo glass transition.

KEY WORDS Ferroelectric Main-Chain Liquid Crystalline Polymer / Dielectric Relaxation / Goldstone Mode / Glass Transition /
\end{abstract}

Main chain liquid crystalline polymers generally consist of alternate sequences of rigid mesogenic group and flexible spacer groups. ${ }^{1}$ The mesogenic group plays a primary role in forming the mesophase structure as in low molecular weight liquid crystals. The flexible group is introduced to control the mesophase structure as well as transition temperature between different mesophases. Its role is more essential in liquid crystalline polymers than in low molecular weight liquid crystals.

Watanabe and coworkers ${ }^{2-6}$ synthesized the BB- $n$ polyesters in which biphenyldicarboxylic acid (mesogen) is connected with alkylene spacer of carbon number $n$. These polyesters form smectic mesophases. Evenmembered BB- $n$ forms a smectic $\mathrm{A}\left(\mathrm{S}_{\mathrm{A}}\right)$ phase while the odd-membered one yields a smectic $\mathrm{CA}\left(\mathrm{S}_{\mathrm{CA}}\right)$ phase. ${ }^{2}$ In the $S_{C A}$ phase, mesogenic groups are tilted to the layer normal like in the conventional smectic $\mathrm{C}$ phase but the tilt direction is opposite in neighboring layers. Such an odd-even parity effect is characteristic of main-chain liquid crystalline polymers and results from the conformational constraint in that the spatial arrangement of the mesogenic groups is strongly confined by the conformation of the intervening methylene groups.

The introduction of branched methyl group into the even-numbered spacer alters the $S_{A}$ phase to the $S_{C}$ phase. ${ }^{3,5}$ More interestingly, if a methyl group is introduced so that the attached carbon becomes asymmetric, one obtains the chiral smectic $\mathrm{C}\left(\mathrm{S}_{\mathrm{C}}{ }^{*}\right)$ phase, which possesses a symmetry required to exhibit a spontaneous polarization leading to an appearance of ferroelectricity.

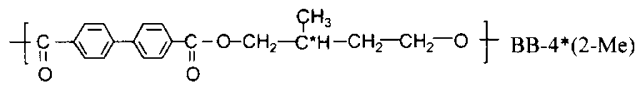

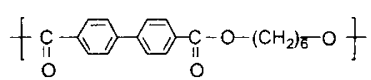

$$
\begin{aligned}
& \text { BB-6 }
\end{aligned}
$$

They synthesized copolymers of BB- $4 *(2-\mathrm{Me})$ and BB- 6 over the entire range of composition and found that an equimolar copolymer has the broadest temperature range of $\mathrm{S}_{\mathrm{C}} *$ phase. They determined the temperature dependence of helical pitch, tilt angle and ferroelectric polarization in the $\mathrm{S}_{\mathrm{C}}$ * phase.

This work undertook dielectric relaxation measurements for a BB- $4^{*}(2-\mathrm{Me}) / \mathrm{BB}-6$ copolymer to substantiate its ferroelectricity and characterize the nature of molecular motion over the broad range of frequency and temperature.

\section{EXPERIMENTAL}

The BB- $4 *(2-\mathrm{Me}) / \mathrm{BB}-6$ sample was synthesized by melt transesterification of dimethyl $p, p^{\prime}$-bibenzoate and an equimolar mixture of $(S)$-2-methylbutanediol and hexanediol. ${ }^{4}$ The degree of polymerization of the product was determined to be $5-6$ by gel permeation choromatography measurement (HLC-8020, Tosoh) using chloroform as solvent. The sample was sandwiched between a pair of ITO-coated glass substrates using a fused silica fiber $10 \mu \mathrm{m}$ in diameter as spacer. Shear deformation was applied through the glass substrates so that the molecules were oriented parallel to the substrates and the smectic layers were aligned parallel to the thickness direction. The area of electrode was $5 \times 5 \mathrm{~mm}^{2}$.

The complex permittivity $\varepsilon^{*}=\varepsilon^{\prime}-i \varepsilon^{\prime \prime}$ was measured over a $10 \mathrm{mHz}-10 \mathrm{MHz}$ frequency range from $-150^{\circ} \mathrm{C}$ to $150^{\circ} \mathrm{C}$ using a laboratory-made dielectric spectrometer and impedance analyzer HP4192A (Hewlett-Packard). In addition to conventional dielectric measurements, a dc bias field was superimposed on a sinusoidal electric field to examine its effects on molecular motion. 


\section{RESULTS AND DISCUSSION}

Figure 1 shows a 3-D representation of the frequency and temperature spectra of $\log \varepsilon^{\prime}$ and $\log \varepsilon^{\prime \prime}$ for $\mathrm{BB}-4 *(2$ $\mathrm{Me}) / \mathrm{BB}-6$. One finds three relaxation processes designated as $\alpha, \beta$, and $\gamma$ in the order of decreasing temperature. In the high temperature range, both $\varepsilon^{\prime}$ and $\varepsilon^{\prime \prime}$ increase with decreasing frequency due to dc conduction and electrode polarization. These were removed in a manner as described later to obtain purely dielectric spectra.

The phase sequences of BB- 4 (2-Me)/BB-6 are represented in Figure 2 in terms of dielectric temperature spectra obtained during cooling at a fixed frequency of $10 \mathrm{~Hz}$. The X-ray, DSC, and optical microscopic observations of this copolymer revealed a phase transition from $\mathrm{S}_{\mathrm{A}}$ to $\mathrm{S}_{\mathrm{C}}{ }^{*}$ at $140^{\circ} \mathrm{C}$. An isotropization of $\mathrm{S}_{\mathrm{A}}$ occurs at around $170^{\circ} \mathrm{C}$. Thus the $\alpha$ process around $100^{\circ} \mathrm{C}$ can be naturally attributed to the Goldstone mode inherent to ferroelectric liquid crystals. ${ }^{6,7}$ The copolymer shows a broad DSC peak at around $70^{\circ} \mathrm{C}$ on cooling. This may be attributed to crystallization, but the degree of crystallinity is negligibly small compared with that of homopolymers. ${ }^{4}$ Thus the $\mathrm{S}_{\mathrm{C}}{ }^{*}$ phase remains in a non-crystalline state below $70^{\circ} \mathrm{C}$. Although Goldstone motion is frozenin below $60^{\circ} \mathrm{C}$, the copolymer still undergoes active motion as demonstrated by additional relaxation processes at lower temperatures. The combined spectra of $\beta$ and $\gamma$ processes shown in Figure 2 look similar to the primary and secondary relaxation processes associated with glass transition ${ }^{8}$ in non-crystalline polymers. The following examines these relaxation processes in detail on the basis of frequency spectra.

\section{$S_{A}-S_{C} *$ Transition}

The $\alpha$-process is much obscured by dc conduction and electrode polarization. To subtract these contributions, we fitted the observed spectra to the following empirical function

$$
\varepsilon^{*}=\varepsilon_{\infty}+\frac{\Delta \varepsilon}{\left\{1+(i \omega \tau)^{\hat{\beta}}\right\}^{\prime \prime}}+\frac{1}{i \omega / \sigma+(i \omega)^{1-r} / \varepsilon_{\mathrm{el}}}
$$

The first and second terms on the right comprise Havriliak-Negami $(\mathrm{H}-\mathrm{N})$ function, ${ }^{9}$ where $\varepsilon_{\infty}$ is the instantaneous permittivity, $\Delta \varepsilon$ relaxation strength, $\tau$ relaxation time, and $\alpha$ and $\beta$ parameters expressing the distribution of relaxation time. In the third term, $\sigma$ is the dc conductivity and $\varepsilon_{\mathrm{el}}$ and $\gamma$ are parameters expressing electrode polarization. ${ }^{10}$ Exemplifying results are shown in Figure 3 where open and filled circles are observed $\varepsilon^{\prime}$ and $\varepsilon "$, respectively, and the solid curves represent fitted spectra. Removing the contribution from the third term, we obtain purely dielectric spectra as shown by dashed curves.

Among quantities determined as best-fit parameters, $\Delta \varepsilon$ and $\log f_{\mathrm{m}}$ are plotted in Figure 4 as a function of temperature. Here $f_{\mathrm{m}}$ is the relaxation frequency given by $1 /$ $2 \pi \tau$ which is nearly equal to the frequency where $\varepsilon$ " becomes a maximum. At $140^{\circ} \mathrm{C}$, both peak due to transition from the $\mathrm{S}_{\mathrm{A}}$ phase to the $\mathrm{S}_{\mathrm{C}}$ * phase. As temperature decreases, $\Delta \varepsilon$ first increases until $100^{\circ} \mathrm{C}$ is reached and then decreases to become undetectable below $60^{\circ} \mathrm{C}$,
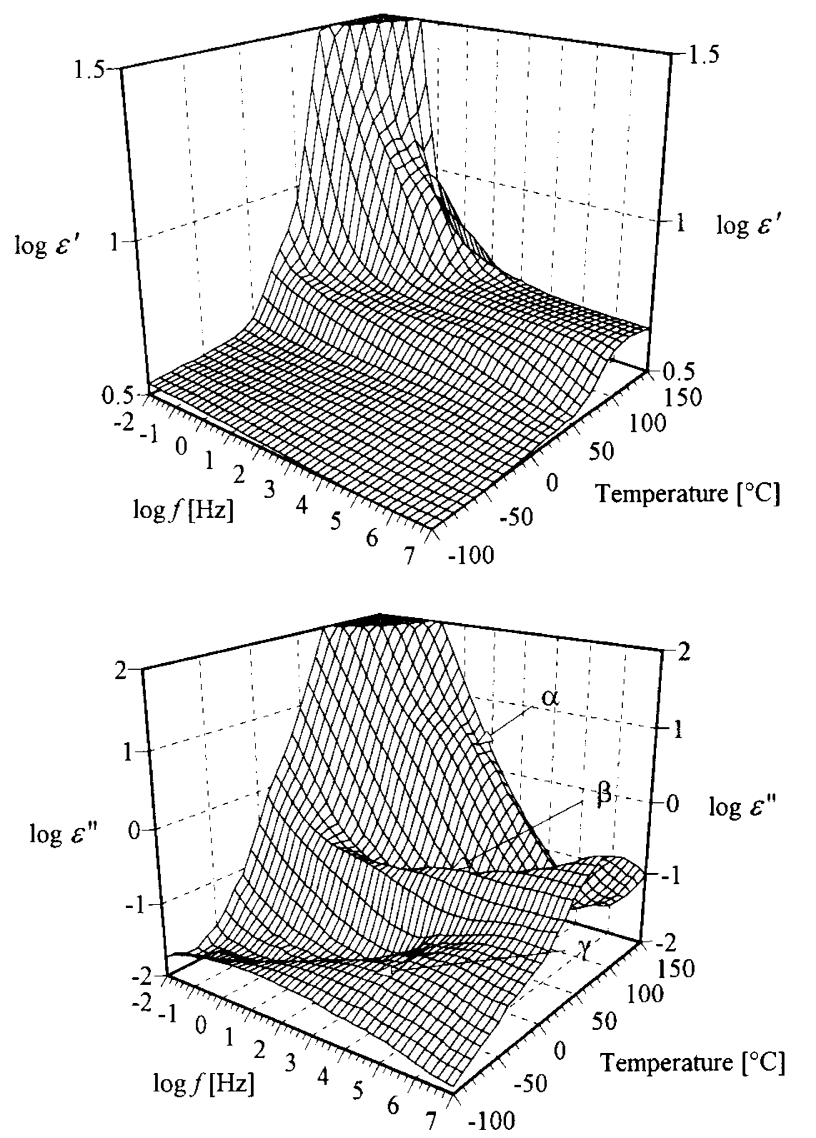

Figure 1. 3-D representation of dielectric frequency and temperature spectra for $\mathrm{BB}-4^{*}(2-\mathrm{Me}) / \mathrm{BB}-6$.

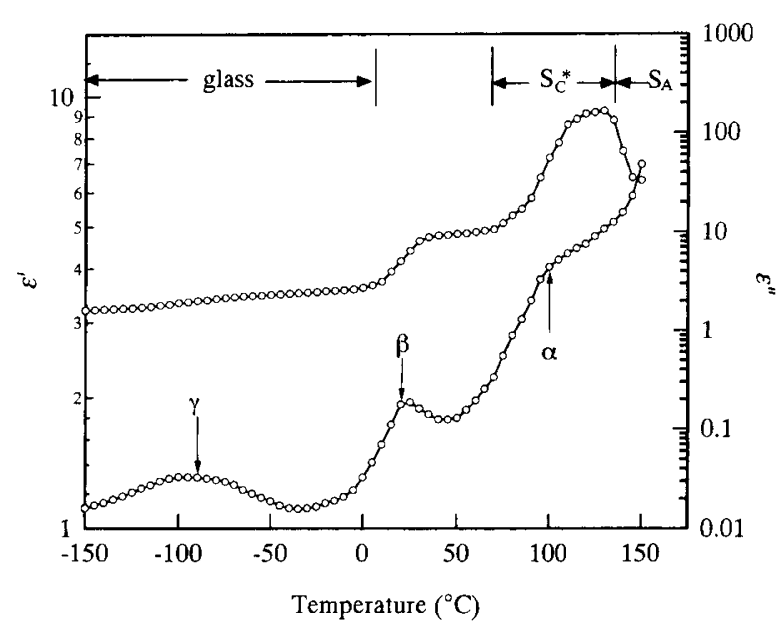

Figure 2. Temperature spectra of $\varepsilon^{\prime}$ and $\varepsilon^{\prime \prime}$ for $\mathrm{BB}-4^{*}(2-\mathrm{Me}) / \mathrm{BB}-6$ at $10 \mathrm{~Hz}$.

whereas $\log f_{\mathrm{m}}$ decreases continuously. Referring to conventional low-molecular weight ferroelectric liquid crystals, this process can be assigned to Goldstone mode associated with fluctuations of the azimuthal angle of tilted molecules in the $\mathrm{S}_{\mathrm{C}}$ * layers forming a helical structure. . $^{71,12}$

To confirm this assignment, we made dielectric measurements in the presence of dc bias field $E_{\mathrm{b}}$. Figure 5 shows the results of measurements at $137^{\circ} \mathrm{C}$ which is 


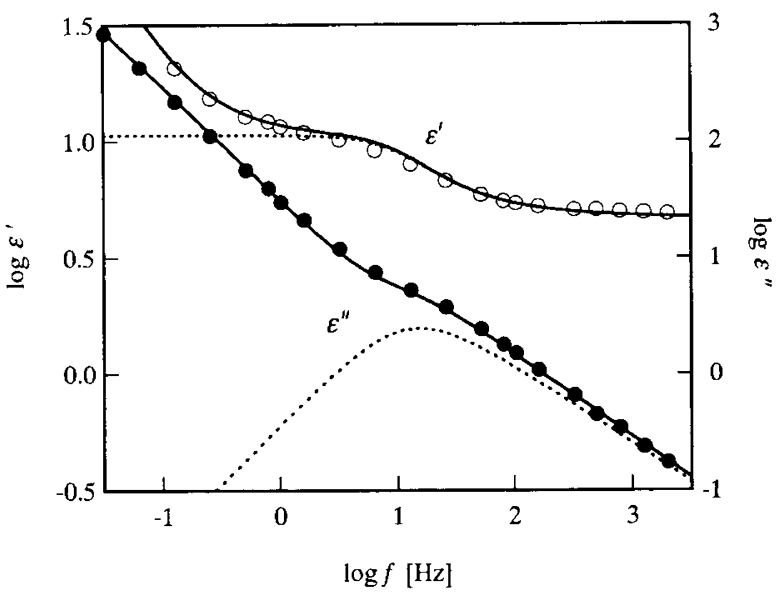

Figure 3. Observed and fitted dielectric frequency spectra associated with Goldstone mode for $\mathrm{BB}-4^{*}(2-\mathrm{Me}) / \mathrm{BB}-6$ at $120^{\circ} \mathrm{C}$.

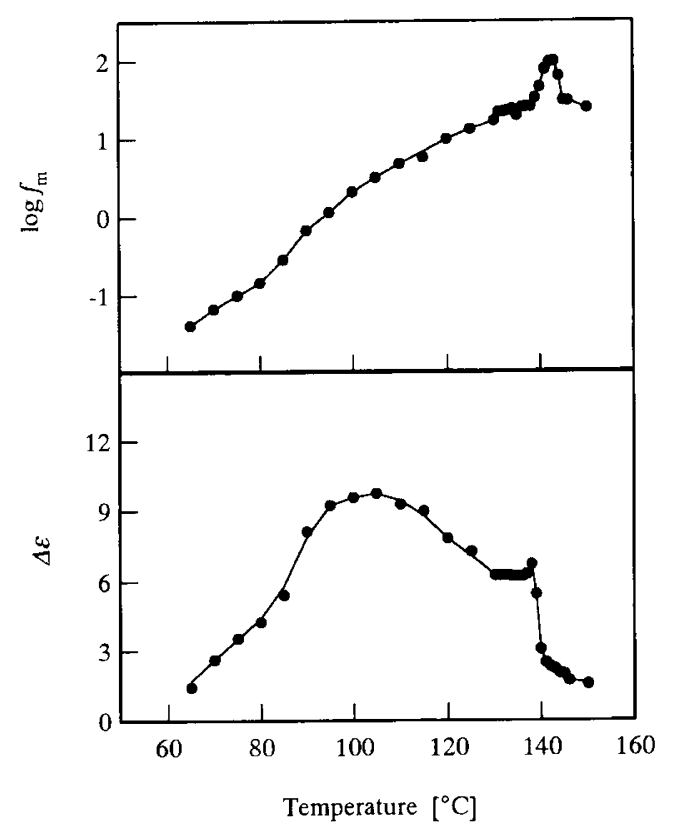

Figure 4. Temperature variation of dielectric relaxation frequency $f_{\mathrm{m}}$ and relaxation strength $\Delta \varepsilon$ associated with Goldstone mode for $\mathrm{BB}-4 *(2-\mathrm{Me}) / \mathrm{BB}-6$.

just below the $\mathrm{S}_{\mathrm{A}}-\mathrm{S}_{\mathrm{C}}$ * transition point. In this figure, contributions from dc conduction and electrode polarization have been removed. The $\alpha$-process is markedly diminished as $E_{\mathrm{b}}$ is imposed, because the bias field causes unwinding of the helical structure inherent to the $\mathrm{S}_{\mathrm{C}}$ * phase to suppress the azimuthal fluctuations. ${ }^{13}$

The bias field dependence of the $\alpha$-process is summarized in Figure 6 where relaxation strength $\Delta \varepsilon$ is plotted against $E_{\mathrm{b}}$. As temperature decreases, $\Delta \varepsilon$ starts to decrease at higher $E_{\mathrm{b}}$ after showing a peak. Such peaking suggests that unwinding of helical structure needs a certain critical field. Although the $\alpha$-process almost disappears above an $E_{\mathrm{b}}$ of $2.5 \mathrm{MVm}^{-1}$, there remains a weak relaxation process at slightly higher frequency. Referring again to conventional ferroelectric liquid crystals, this process can be assigned to the soft mode associated with tilt angle fluctuation. Parallel with this assignment, anomalous $f_{\mathrm{m}}$ and $\Delta \varepsilon$ appeared at $140^{\circ} \mathrm{C}$ in Figure 4 can

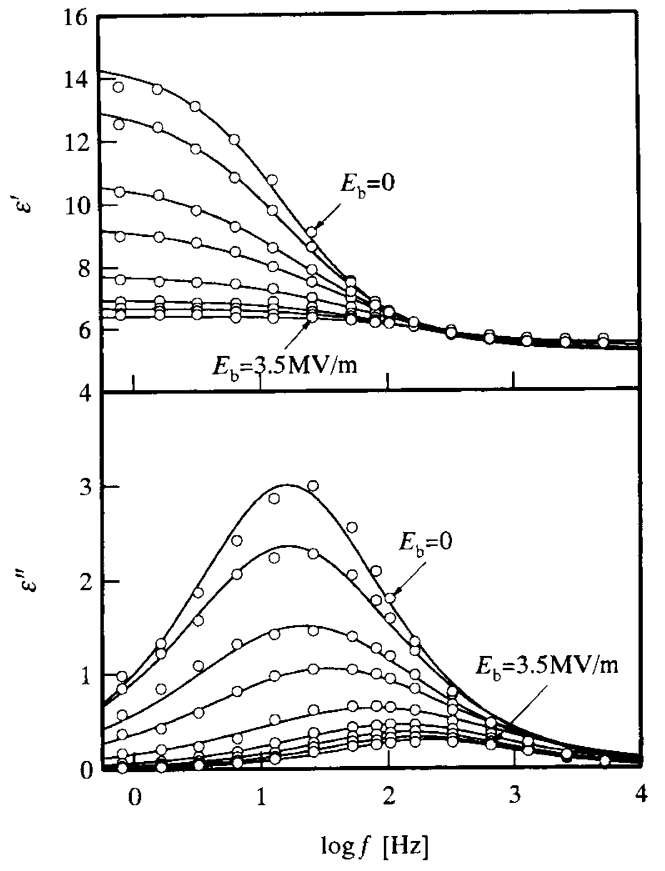

Figure 5. Bias-field dependence of dielectric relaxation frequency spectra associated with Goldstone mode for $\mathrm{BB}-4 *(2-\mathrm{Me}) / \mathrm{BB}-6$ at $137^{\circ} \mathrm{C}$.

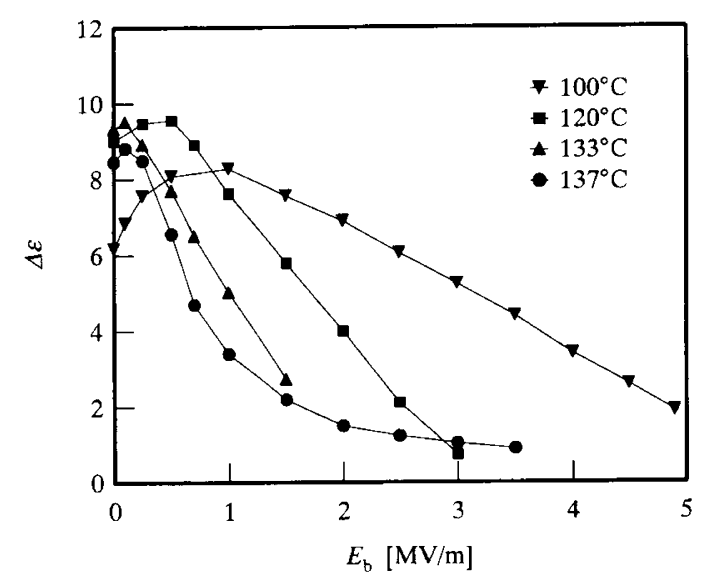

Figure 6. Bias-field dependence of the dielectric relaxation strength $\Delta \varepsilon$ associated with Goldstone mode for BB- $4^{*}(2-\mathrm{Me}) / \mathrm{BB}-6$.

be interpreted in terms of softening of such a mode.

The dielectric relaxation strength $\Delta \varepsilon_{\mathrm{G}}$ and relaxation time $\tau_{\mathrm{G}}$ associated with Goldstone mode are given as follows ${ }^{14}$

$$
\begin{gathered}
\Delta \varepsilon_{\mathrm{G}}=\frac{P_{\mathrm{s}}{ }^{2}}{2 \varepsilon_{0} K q_{0}{ }^{2}} \\
\tau_{\mathrm{G}}=\frac{\gamma}{K q_{0}^{2}}
\end{gathered}
$$

where $K$ is the effective elastic constant, $\gamma$ effective rotational viscosity, $q_{0}$ wave number of the helical structure, $\varepsilon_{0}$ vacuum permittivity and $P_{\mathrm{s}}$ spontaneous polarization. From Figure 4, we obtain $\Delta \varepsilon_{G}=6$ and $\tau_{G}=8 \times 10^{-3}$ s at 130 ${ }^{\circ} \mathrm{C}, 10^{\circ} \mathrm{C}$ below the transition point. The spectroscopic observation of reflection bands has yielded $q_{0}=1.3 \times 10^{7}$ $\mathrm{rad} \mathrm{m}^{-1}$ for an equimolar BB- $4 *(2-\mathrm{Me}) / \mathrm{BB}-6$ being less 


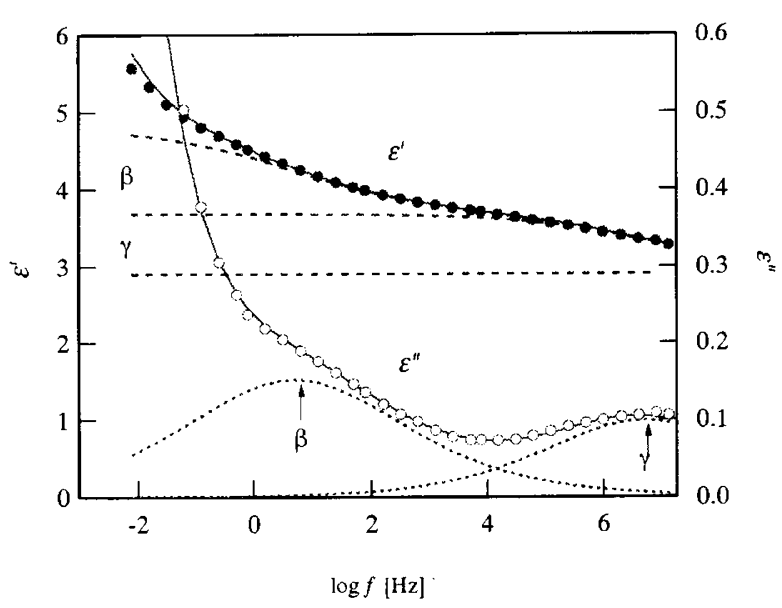

Figure 7. Decomposition of dielectric relaxation frequency spectra associated with $\beta$ and $\gamma$-processes for BB- $4^{*}(2-\mathrm{Me}) / \mathrm{BB}-6$ at $25^{\circ} \mathrm{C}$.

dependent on temperature. The spontaneous polarization $P_{\mathrm{s}}$ was determined to be $150 \mu \mathrm{C} \mathrm{m}^{-2}$ based on ferroelectric switching measurements. Using these results, we obtain $K=1.3 \mathrm{pN}$ and $\gamma=1.8 \mathrm{~Pa}$ s. It is interesting to note that the value of $K$ is similar whereas $\gamma$ is $10-100$ times larger compared to low-molecular-weight ferroelectric liquid crystals. ${ }^{15,16}$ This means that the methylene chains connecting smectic layers BB- $4 *(2-\mathrm{Me}) / \mathrm{BB}-6$ causes increase in viscosity but does not affect the elastic constant.

\section{Glass Transition}

At low temperatures where the Goldstone mode motion is frozen-in, there exist two additional relaxation processes $\beta$ and $\gamma$. The filled and open circles in Figure 7 show the frequency spectra of $\varepsilon^{\prime}$ and $\varepsilon^{\prime \prime}$, respectively, at $25^{\circ} \mathrm{C}$ associated with these processes. As the $\beta$-process is overtaken by $\mathrm{dc}$ conduction at low frequency, we made curve-fitting using eq 1 with two $\mathrm{H}-\mathrm{N}$ functions. The solid curves show the fitted spectra and the dashed curves express resolved $\beta$ and $\gamma$ processes. Figure 8 shows plots of $\log f_{\mathrm{m}} v s$. reciprocal absolute temperature $1 / T$ (transition map) for these processes as well as Goldstone and soft mode processes during heating and cooling. The $\gamma$-process is of Arrhenius-type whereas the $\beta$ process obeys a Vogel-Fulcher $(\mathrm{V}-\mathrm{F})$ function

$$
f_{\mathrm{m}}=f_{\infty} \exp \left(-\frac{B}{T-T_{0}}\right) .
$$

Here $T_{0}$ is the temperature where $f_{\mathrm{m}}$ becomes $0, f_{\infty}$ is the relaxation frequency at very high temperatures and $B$ is a constant. Fitting the observed relaxation frequencies to this function yielded $T_{0}=259 \mathrm{~K}, f_{\infty}=10^{14.1} \mathrm{~Hz}$ and $B=$ $1234 \mathrm{~K}$. The activation energy for the Arrhenius-type $\gamma$ process is estimated to be $42 \mathrm{~kJ} \mathrm{~mol}^{-1}$ from the slope of $\log f_{\mathrm{m}}$ vs. $1 / T$ plot.

The $\mathrm{V}-\mathrm{F}$ function is characteristic of a cooperative process and generally applies to glass transition. A combination of $\mathrm{V}-\mathrm{F}$ type $\beta$ process and Arrehenius type $\gamma$ process that merge at $80^{\circ} \mathrm{C}$ is commonly observed for noncrystalline polymers. BB- $4 *(2-\mathrm{Me}) / \mathrm{BB}-6$ copolymer may thus undergo glass transition. $T_{\mathrm{g}}$ is evaluated to be $15^{\circ} \mathrm{C}$ from the temperature where $\log f_{\mathrm{m}}$ becomes -3 . This does not necessarily mean that BB- $4 *(2-\mathrm{Me}) / \mathrm{BB}-6$

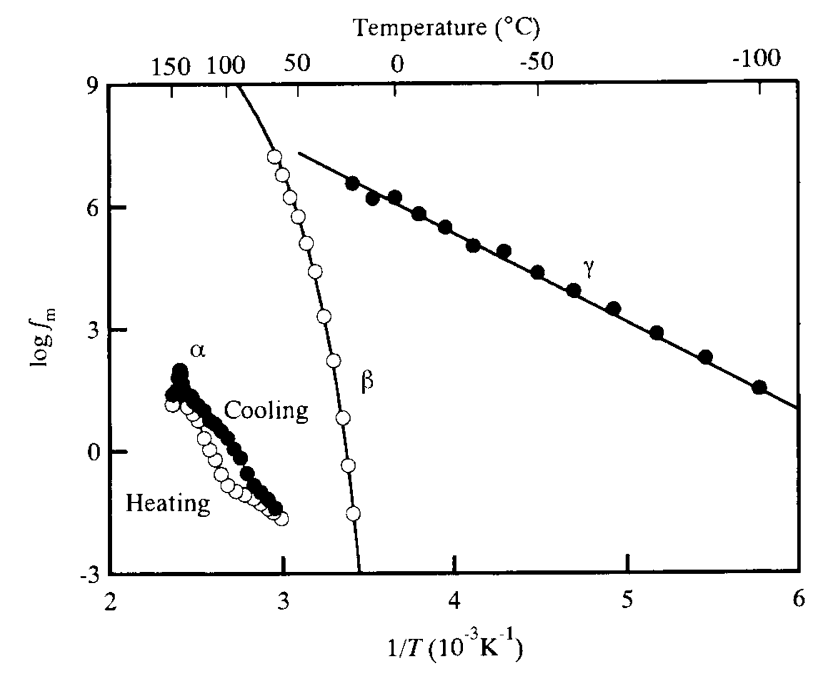

Figure 8. Transition map of BB- $4 *(2-\mathrm{Me}) / \mathrm{BB}-6$.

assumes rubber-like amorphous structure. In fact, it exhibits a ferroelectric liquid crystalline phase $S_{C}{ }^{*}$ possessing layered structures at $60-140^{\circ} \mathrm{C}$. The Goldstone mode motion characteristic of such a phase freezes at 60 ${ }^{\circ} \mathrm{C}$. Below this temperature, BB- $4 *(2-\mathrm{Me}) / \mathrm{BB}-6$ is expected to take on a structure with higher symmetry. Nevertheless, it must possess certain disorder allowing a cooperative motion that gives rise to glass transition. ${ }^{17}$ Details of such a motion remain to be pursued.

In conclusion, BB- $4 *(2-\mathrm{Me}) / \mathrm{BB}-6$ is a ferroelectric liquid crystalline polymer undergoing Goldstone and soft mode motion as well as glass transition. Goldstone mode motion is confirmed by its suppression by dc bias field. Glass transition is demonstrated by cooperative motion that freezes rapidly at $15^{\circ} \mathrm{C}$ obeying a V-F function. The coexistence of Goldstone mode and glass transition shows that $\mathrm{BB}-4^{*}(2-\mathrm{Me}) / \mathrm{BB}-6$ possesses both orientational order and spatial disorder. In the $\mathrm{S}_{\mathrm{C}}$ * phase, smectic layers are connected by methylene chains. Such constraint results in larger inter-layer rotational viscosity, but does not suppress ferroelectricity. We observed relatively fast ferroelectric polarization switching phenomena for BB-4*(2-Me)/BB-6.

\section{REFERENCES}

1. A. Ciferri, W. R. Krigbaum, and W. R. Meyer, "Polymer Liquid Crystals", Academic Press, New York, N.Y., 1982.

2. J. Watanabe, and M. Hayashi, Macromolecules, 21, 278 (1988).

3. J. Watanabe, M. Hayashi, S. Kinoshita, and T. Niori, Polym. J., 24, 597 (1992).

4. J. Watanabe, M. Hayashi, A. Morita, and M. Tokita, Macromolecules, 28, 8073 (1995).

5. Y. Nakata, M.Hayashi, M. Tokita, K. Osada, and J. Watanabe, High Perform. Polym., 10, 121 (1998).

6. K. Hiraoka, Y. Sugano, Y. Sugano, K. Monzen, Y. Uematsu, M. Tokita, J. Watanabe, and T. Furukawa, Mol. Cryst. Liq. Cryst., 299, 229 (1997).

7. R. Blinc, and B. Zekš, Phys. Rev. A, 18, 740 (1978).

8. M. Tokita, K. Osada, and J. Watanabe, Polym. J., 30, 589 (1998).

9. S. Havriliak, and S. Negami, J. Polym. Sci., C14, 99 (1966).

10. M. Imura, and T. Furukawa, Jpn. J. Appl. Phys., 36, 1119 
(1997).

11. S. U. Vallerian, F. Kremer, T. Geelhaar, and A. E. Wächtler, Phys. Rev. A, 42, 2482 (1990).

12. F. Kremer, S. U. Vallerian, R. Zentel, and H. Kapitza, Macromol. Chem., Macromol. Symp., 44, 127 (1991).

13. H. Xu, J. K. Vij, A. Rappaport, and N. A. Clark, Phys. Rev., 79, 249 (1997).

14. T. Carlsson, B. Žekš, C. Filipič, and A. Levstik, Phys. Rev. A,
42, 877 (1990).

15. H. Takezoe, K. Kondo, S. Abe, K. Miyasato, T. Tsuchida, A. Fukuda, and E. Kuze, Ferroelectrics, 58, 55 (1984).

16. A. Fukuda, and H. Takezoe, "Structure and Properties of Ferroelectric Liquid Crystals", Corona Pub., Tokyo, 1990, p 315.

17. Y. González, B. Palacios, M. A. P. Jubindo, M. R. de la Fuente, and J. L. Serrano, Phys. Rev. E, 52, 5764 (1995). 